\title{
A SUM FORM FUNCTIONAL EQUATION RELATED TO VARIOUS ENTROPIES IN INFORMATION THEORY
}

\author{
Prem Nath and Dhiraj Kumar Singh
}

University of Delhi, India

\begin{abstract}
The general solutions of a sum form functional equation have been investigated. The relevance of these solutions in relation to various entropies in information theory has been emphasized.
\end{abstract}

\section{INTRODUCTION}

For $n=1,2, \ldots$ let

$$
\Gamma_{n}=\left\{\left(p_{1}, \ldots, p_{n}\right): p_{i} \geq 0, i=1, \ldots, n ; \sum_{i=1}^{n} p_{i}=1\right\}
$$

denote the set of all $n$-component discrete complete probability distributions with nonnegative elements.

The Shannon entropy [5] of a probability distribution $\left(p_{1}, \ldots, p_{n}\right) \in \Gamma_{n}$ is defined as

$$
H_{n}\left(p_{1}, \ldots, p_{n}\right)=-\sum_{i=1}^{n} p_{i} \log _{2} p_{i}
$$

where $H_{n}: \Gamma_{n} \rightarrow \mathbb{R}, n=1,2, \ldots, \mathbb{R}$ denotes the set of all real numbers and $0 \log _{2} 0=0$. A generalization of the Shannon entropy (A) with which we shall be concerned in this paper is (with $H_{n}^{\alpha}: \Gamma_{n} \rightarrow \mathbb{R}, n=1,2, \ldots$ )

$$
H_{n}^{\alpha}\left(p_{1}, \ldots, p_{n}\right)=\left(1-2^{1-\alpha}\right)^{-1}\left(1-\sum_{i=1}^{n} p_{i}^{\alpha}\right)
$$

2000 Mathematics Subject Classification. 39B52, 39B82.

Key words and phrases. Sum form functional equation, additive function, multiplicative function, the Shannon entropy, the nonadditive entropies of degree $\alpha$. 
where $\alpha>0, \alpha \neq 1,0^{\alpha}:=0,1^{\alpha}:=1$ and $\alpha \in \mathbb{R}$. The entropies (B) are known as the nonadditive entropies of degree $\alpha, \alpha>0, \alpha \neq 1$ and are due to Havrda and Charvát [2]. It can be easily seen that

$$
\lim _{\alpha \rightarrow 1} H_{n}^{\alpha}\left(p_{1}, \ldots, p_{n}\right)=-\sum_{i=1}^{n} p_{i} \log _{2} p_{i}=H_{n}\left(p_{1}, \ldots, p_{n}\right) .
$$

Losonczi and Maksa [3] considered the multiplicative type sum form functional equation

$$
\sum_{i=1}^{n} \sum_{j=1}^{m} f\left(p_{i} q_{j}\right)=\sum_{i=1}^{n} f\left(p_{i}\right) \sum_{j=1}^{m} f\left(q_{j}\right)
$$

where $f: I \rightarrow \mathbb{R}, I=\{x \in \mathbb{R}: 0 \leq x \leq 1\},\left(p_{1}, \ldots, p_{n}\right) \in \Gamma_{n}$ and $\left(q_{1}, \ldots, q_{m}\right) \in \Gamma_{m}$. They found the general solutions of (1.1) for all $\left(p_{1}, \ldots, p_{n}\right) \in \Gamma_{n},\left(q_{1}, \ldots, q_{m}\right) \in \Gamma_{m}, n \geq 3, m \geq 3$ being fixed integers.

In their recent research work, Nath and Singh [4] considered the multiplicative type functional equation

$$
\sum_{i=1}^{n} \sum_{j=1}^{m} f\left(p_{i} q_{j}\right)=\sum_{i=1}^{n} g\left(p_{i}\right) \sum_{j=1}^{m} h\left(q_{j}\right)
$$

where $f: I \rightarrow \mathbb{R}, g: I \rightarrow \mathbb{R}, h: I \rightarrow \mathbb{R},\left(p_{1}, \ldots, p_{n}\right) \in \Gamma_{n}$ and $\left(q_{1}, \ldots, q_{m}\right) \in$ $\Gamma_{m}$. They found the general solutions of $(1.2)$ for all $\left(p_{1}, \ldots, p_{n}\right) \in \Gamma_{n}$, $\left(q_{1}, \ldots, q_{m}\right) \in \Gamma_{m}, n \geq 3, m \geq 3$ being fixed integers. During the process of finding these general solutions, they came across the functional equation

$$
\begin{aligned}
\sum_{i=1}^{n} \sum_{j=1}^{m} T\left(p_{i} q_{j}\right)= & \sum_{i=1}^{n} T\left(p_{i}\right) \sum_{j=1}^{m} T\left(q_{j}\right)+(m-n) T(0) \sum_{j=1}^{m} T\left(q_{j}\right) \\
& +m(n-1) T(0)
\end{aligned}
$$

with $T: I \rightarrow \mathbb{R}$; found its general solutions for all $\left(p_{1}, \ldots, p_{n}\right) \in \Gamma_{n},\left(q_{1}, \ldots, q_{m}\right)$ $\in \Gamma_{m}, n \geq 3, m \geq 3$ being fixed integers; and utilized these solutions to find the corresponding solutions of (1.2).

There are several nonmultiplicative type sum form functional equations of which the functional equation (1.2) is just a part. One such functional equations is

$$
\sum_{i=1}^{n} \sum_{j=1}^{m} f\left(p_{i} q_{j}\right)=\sum_{i=1}^{n} g\left(p_{i}\right) \sum_{j=1}^{m} h\left(q_{j}\right)+\sum_{j=1}^{m} f\left(q_{j}\right) .
$$

If the last term on the right hand side of (1.4) is omitted, we obtain (1.2). It is in this sense that (1.2) is a part of (1.4).

The purpose of this paper is to determine the general solutions of (1.4) for all $\left(p_{1}, \ldots, p_{n}\right) \in \Gamma_{n},\left(q_{1}, \ldots, q_{m}\right) \in \Gamma_{m}, n \geq 3, m \geq 3$ being fixed integers and then discuss the importance of these solutions from information-theoretic 
point of view, especially, in connection with the entropies (A) and (B). These two tasks have been done in sections 3 and 4 respectively.

The process of determining the general solutions of (1.4) for all $\left(p_{1}, \ldots, p_{n}\right)$ $\in \Gamma_{n},\left(q_{1}, \ldots, q_{m}\right) \in \Gamma_{m}, n \geq 3, m \geq 3$ being fixed integers, needs determining the general solutions of the functional equation

$$
\begin{aligned}
\sum_{i=1}^{n} \sum_{j=1}^{m} g\left(p_{i} q_{j}\right)= & \sum_{i=1}^{n} g\left(p_{i}\right) \sum_{j=1}^{m} H\left(q_{j}\right) \\
& +\sum_{j=1}^{m} g\left(q_{j}\right)-g(1)+(n-1)(m-1) g(0)
\end{aligned}
$$

for all $\left(p_{1}, \ldots, p_{n}\right) \in \Gamma_{n},\left(q_{1}, \ldots, q_{m}\right) \in \Gamma_{m}, n \geq 3, m \geq 3$ being fixed integers and $g: I \rightarrow \mathbb{R}, H: I \rightarrow \mathbb{R}$ such that $H$ satisfies the condition

$$
H(1)+(m-1) H(0)=1 .
$$

This has been done in section 2 .

\section{The general solutions of Functional EQuation (1.5) WITH CONDITION (1.6)}

Before investigating the general solutions of (1.5), with $H$ satisfying the condition (1.6), we need some definitions and results already available in the literature (see Losonczi and Maksa [3]).

Let $\Delta=\{(x, y): 0 \leq x \leq 1,0 \leq y \leq 1,0 \leq x+y \leq 1\}$. A mapping $a: I \rightarrow \mathbb{R}$ is said to be additive on $I$ if $a(x+y)=a(x)+a(y)$ holds for all $(x, y) \in \Delta$. A mapping $A: \mathbb{R} \rightarrow \mathbb{R}$ is said to be additive on $\mathbb{R}$ if

$$
A(x+y)=A(x)+A(y)
$$

holds for all $x \in \mathbb{R}, y \in \mathbb{R}$. It is known (see Daróczy and Losonczi [1]) that if a mapping $a: I \rightarrow \mathbb{R}$ is additive on $I$, then it has a unique additive extension $A: \mathbb{R} \rightarrow \mathbb{R}$ in the sense that $A: \mathbb{R} \rightarrow \mathbb{R}$ is additive on $\mathbb{R}$ and $A(x)=a(x)$ for all $x \in I$.

Definition 2.1. A mapping $M: I \rightarrow \mathbb{R}$ is said to be multiplicative on $I$ if $M(0)=0, M(1)=1$ and $M(p q)=M(p) M(q)$ for all $p \in] 0,1[, q \in] 0,1[$ where $] 0,1[=\{x \in \mathbb{R}: 0<x<1\}$.

RESUlT 1 ([3]). Let $k \geq 3$ be a fixed integer and $c$ be a given constant. Suppose a mapping $\varphi: I \rightarrow \mathbb{R}$ satisfies the equation

$$
\sum_{i=1}^{k} \varphi\left(p_{i}\right)=c
$$


for all $\left(p_{1}, \ldots, p_{k}\right) \in \Gamma_{k}$. Then there exists an additive mapping $B: \mathbb{R} \rightarrow \mathbb{R}$ such that

$$
\varphi(p)=B(p)-\frac{1}{k} B(1)+\frac{c}{k}
$$

for all $p \in I$.

ReSUlT 2 ([3]). If a mapping $G: I \rightarrow \mathbb{R}$ satisfies the functional equation

$$
\sum_{i=1}^{n} \sum_{j=1}^{m} G\left(p_{i} q_{j}\right)=\sum_{i=1}^{n} G\left(p_{i}\right)+\sum_{j=1}^{m} G\left(q_{j}\right)
$$

for all $\left(p_{1}, \ldots, p_{n}\right) \in \Gamma_{n},\left(q_{1}, \ldots, q_{m}\right) \in \Gamma_{m}, n \geq 3, m \geq 3$ being fixed integers, then $G$ is of the form

$$
G(p)= \begin{cases}G(0)+G(0)(n m-n-m) p+a(p)+D(p, p) & \text { if } 0<p \leq 1 \\ G(0) & \text { if } p=0\end{cases}
$$

where $G(0)$ is an arbitrary real constant; $a: \mathbb{R} \rightarrow \mathbb{R}$ is additive on $\mathbb{R}$; $D: \mathbb{R} \times] 0,1] \rightarrow \mathbb{R}$ is additive in the first variable, $] 0,1]=\{x \in \mathbb{R}: 0<x \leq 1\}$; there exists a mapping $E: \mathbb{R} \times \mathbb{R} \rightarrow \mathbb{R}$, additive in both variables, such that $a(1)=E(1,1)$ and

$$
D(p q, p q)=D(p q, p)+D(p q, q)+E(p, q)
$$

holds for all $p \in] 0,1], q \in] 0,1]$.

From (2.4), it can be easily deduced that

$$
a(1)+D(1,1)=0
$$

as $E(1,1)=a(1)$.

RESUlT 3 ([4]). If a mapping $T: I \rightarrow \mathbb{R}$ satisfies the functional equation (1.3) for all $\left(p_{1}, \ldots, p_{n}\right) \in \Gamma_{n},\left(q_{1}, \ldots, q_{m}\right) \in \Gamma_{m}, n \geq 3, m \geq 3$ being fixed integers, then $T$ is of the form

$$
T(p)=\bar{a}(p)+T(0)
$$

where $\bar{a}: \mathbb{R} \rightarrow \mathbb{R}$ is an additive mapping with

$$
\bar{a}(1)= \begin{cases}-m T(0) & \text { if } T(1)+(m-1) T(0)-1 \neq 0 \\ 1-m T(0) & \text { if } T(1)+(m-1) T(0)-1=0\end{cases}
$$

or

$$
T(p)=M(p)-b(p)+T(0)
$$

where $b: \mathbb{R} \rightarrow \mathbb{R}$ is an additive mapping with $b(1)=m T(0)$ and $M: I \rightarrow \mathbb{R}$ is a nonconstant nonadditive mapping which is also multiplicative in the sense of Definition 2.1.

Now we prove: 
Theorem 2.2. Let $n \geq 3, m \geq 3$ be fixed integers and $g: I \rightarrow \mathbb{R}$, $H: I \rightarrow \mathbb{R}$ be mappings which satisfy the functional equation (1.5) for all $\left(p_{1}, \ldots, p_{n}\right) \in \Gamma_{n}$ and $\left(q_{1}, \ldots, q_{m}\right) \in \Gamma_{m}$. Suppose $H: I \rightarrow \mathbb{R}$ also satisfies the condition (1.6). Then any general solution of (1.5) is of the form

$$
\left\{\begin{aligned}
g(p) & =g(0)+[g(1)-g(0)] p+a(p)+D(p, p) & & \text { if } 0<p \leq 1 \\
& =g(0) & & \text { if } p=0 \\
H(p) & =b_{1}(p)-\frac{1}{m} b_{1}(1)+\frac{1}{m} & &
\end{aligned}\right.
$$

with $a: \mathbb{R} \rightarrow \mathbb{R}$ and $D: \mathbb{R} \times] 0,1] \rightarrow \mathbb{R}$ as explained in Result 2 or

$$
\left\{\begin{array}{l}
g(p)=b_{2}(p)+g(0) \\
H \text { any real-valued function satisfying (1.6) }
\end{array}\right.
$$

with

$$
b_{2}(1)=-n g(0)
$$

or

$(2.9)\left\{\begin{array}{l}g(p)=-\left[b_{3}(1)+n g(0)\right] M(p)+b_{3}(p)+g(0), b_{3}(1)+n g(0) \neq 0 \\ H(p)=M(p)-b(p)+H(0)\end{array}\right.$

with

$$
b(1)=m H(0)
$$

where $b: \mathbb{R} \rightarrow \mathbb{R}, b_{i}: \mathbb{R} \rightarrow \mathbb{R}(i=1,2,3)$ are additive mappings and $M: I \rightarrow \mathbb{R}$ is a nonconstant nonadditive mapping which is multiplicative in the sense of Definition 2.1.

Proof. Let us write (1.5) in the form

$$
\begin{aligned}
& \sum_{i=1}^{n}\left\{\sum_{j=1}^{m} g\left(p_{i} q_{j}\right)-g\left(p_{i}\right) \sum_{j=1}^{m} H\left(q_{j}\right)-p_{i} \sum_{j=1}^{m} g\left(q_{j}\right)\right\} \\
& \quad=-g(1)+(n-1)(m-1) g(0) .
\end{aligned}
$$

By Result 1 , there exists a mapping $A: \mathbb{R} \times \Gamma_{m} \rightarrow \mathbb{R}$, additive in the first variable such that

$$
\begin{gathered}
\sum_{j=1}^{m} g\left(p q_{j}\right)-g(p) \sum_{j=1}^{m} H\left(q_{j}\right)-p \sum_{j=1}^{m} g\left(q_{j}\right) \\
=A\left(p ; q_{1}, \ldots, q_{m}\right)-\frac{1}{n} A\left(1 ; q_{1}, \ldots, q_{m}\right) \\
+\frac{1}{n}\{-g(1)+(n-1)(m-1) g(0)\}
\end{gathered}
$$


for all $p \in I$. The substitution $p=0$ in (2.11) and the use of the fact that $A\left(0 ; q_{1}, \ldots, q_{m}\right)=0$ gives

$$
A\left(1 ; q_{1}, \ldots, q_{m}\right)=-g(1)-(n+m-1) g(0)+n g(0) \sum_{j=1}^{m} H\left(q_{j}\right)
$$

From (2.11) and (2.12), after performing necessary calculation work, we obtain

$$
\begin{aligned}
& \sum_{j=1}^{m} g\left(p q_{j}\right)-[g(p)-g(0)] \sum_{j=1}^{m} H\left(q_{j}\right)-p \sum_{j=1}^{m} g\left(q_{j}\right) \\
& \quad=A\left(p ; q_{1}, \ldots, q_{m}\right)+m g(0)
\end{aligned}
$$

for all $p \in I$.

Let $\left(r_{1}, \ldots, r_{m}\right) \in \Gamma_{m}$ be any probability distribution. Putting $p=$ $r_{1}, \ldots, r_{m}$ successively in (2.13), adding the resulting $m$ equations and using the additivity of $A: \mathbb{R} \times \Gamma_{m} \rightarrow \mathbb{R}$ in the first variable, it follows that

$$
\begin{aligned}
& \sum_{t=1}^{m} \sum_{j=1}^{m} g\left(r_{t} q_{j}\right)-\left[\sum_{t=1}^{m} g\left(r_{t}\right)-m g(0)\right] \sum_{j=1}^{m} H\left(q_{j}\right)-\sum_{j=1}^{m} g\left(q_{j}\right) \\
& \quad=A\left(1 ; q_{1}, \ldots, q_{m}\right)+m^{2} g(0) .
\end{aligned}
$$

From (2.12) and (2.14),

$$
\begin{aligned}
& \sum_{t=1}^{m} \sum_{j=1}^{m} g\left(r_{t} q_{j}\right)+g(1)-\left(m^{2}-m-n+1\right) g(0) \\
& \quad=\left[\sum_{t=1}^{m} g\left(r_{t}\right)+(n-m) g(0)\right] \sum_{j=1}^{m} H\left(q_{j}\right)+\sum_{j=1}^{m} g\left(q_{j}\right) .
\end{aligned}
$$

The left hand side of (2.15) is symmetric in $r_{t}$ and $q_{j}, t=1, \ldots, m, j=$ $1, \ldots, m$. So, the right hand side of (2.15) must also be symmetric in $r_{t}$ and $q_{j}$. This gives us the equation

$$
\begin{aligned}
& {\left[\sum_{t=1}^{m} g\left(r_{t}\right)+(n-m) g(0)\right]\left[\sum_{j=1}^{m} H\left(q_{j}\right)-1\right]} \\
& \quad=\left[\sum_{j=1}^{m} g\left(q_{j}\right)+(n-m) g(0)\right]\left[\sum_{t=1}^{m} H\left(r_{t}\right)-1\right] .
\end{aligned}
$$

CASE 1. $\sum_{j=1}^{m} H\left(q_{j}\right)-1$ vanishes identically on $\Gamma_{m}$. This means that

$$
\sum_{j=1}^{m} H\left(q_{j}\right)=1
$$


for all $\left(q_{1}, \ldots, q_{m}\right) \in \Gamma_{m}$. By Result 1, there exists an additive mapping $b_{1}: \mathbb{R} \rightarrow \mathbb{R}$ such that

$$
H(p)=b_{1}(p)-\frac{1}{m} b_{1}(1)+\frac{1}{m}
$$

for all $p \in I$. From (1.5) and (2.17), we obtain the equation

$$
\sum_{i=1}^{n} \sum_{j=1}^{m} g\left(p_{i} q_{j}\right)=\sum_{i=1}^{n} g\left(p_{i}\right)+\sum_{j=1}^{m} g\left(q_{j}\right)-g(1)+(n-1)(m-1) g(0)
$$

valid for all $\left(p_{1}, \ldots, p_{n}\right) \in \Gamma_{n},\left(q_{1}, \ldots, q_{m}\right) \in \Gamma_{m}, n \geq 3, m \geq 3$ being fixed integers. Define the mapping $G: I \rightarrow \mathbb{R}$ as

$$
G(x)=g(x)-[g(1)-(n-1)(m-1) g(0)] x
$$

for all $x \in I$. Then (2.19) reduces to the equation (2.2). By Result $2, G$ is of the form (2.3) with $a: \mathbb{R} \rightarrow \mathbb{R}$ and $D: \mathbb{R} \times] 0,1] \rightarrow \mathbb{R}$ as described in Result 2 . Also, from (2.20), we have

$$
G(0)=g(0)
$$

From $(2.20),(2.21)$ and $(2.3)$, it follows that

$$
g(p)= \begin{cases}g(0)+[g(1)-g(0)] p+a(p)+D(p, p) & \text { if } 0<p \leq 1 \\ g(0) & \text { if } p=0\end{cases}
$$

Equations (2.22) and (2.18), taken together, constitute the solution (2.6) of equation (1.5) with $a: \mathbb{R} \rightarrow \mathbb{R}$ and $D: \mathbb{R} \times] 0,1] \rightarrow \mathbb{R}$ as described in Result 2 . Notice that (2.18) implies (1.6).

CASE 2. $\sum_{j=1}^{m} H\left(q_{j}\right)-1$ does not vanish identically on $\Gamma_{m}$. Then, there exists a probability distribution $\left(q_{1}^{*}, \ldots, q_{m}^{*}\right) \in \Gamma_{m}$ such that

$$
\sum_{j=1}^{m} H\left(q_{j}^{*}\right)-1 \neq 0 \text {. }
$$

Putting $q_{j}=q_{j}^{*}, j=1, \ldots, m$ in (2.16) and performing necessary calculations, it follows that

$$
\sum_{t=1}^{m} g\left(r_{t}\right)=\lambda\left[\sum_{t=1}^{m} H\left(r_{t}\right)-1\right]-(n-m) g(0)
$$

where

$$
\lambda=\left[\sum_{j=1}^{m} H\left(q_{j}^{*}\right)-1\right]^{-1}\left[\sum_{j=1}^{m} g\left(q_{j}^{*}\right)+(n-m) g(0)\right] .
$$

CAse 2.1. $\lambda=0$. 
In this case, (2.24) reduces to the equation

$$
\sum_{t=1}^{m} g\left(r_{t}\right)=(m-n) g(0)
$$

valid for all $\left(r_{1}, \ldots, r_{m}\right) \in \Gamma_{m}$. By Result 1 , there exists an additive mapping $b_{2}: \mathbb{R} \rightarrow \mathbb{R}$ such that

$$
g(p)=b_{2}(p)-\frac{1}{m} b_{2}(1)+\frac{1}{m}(m-n) g(0)
$$

for all $p \in I$. The substitution $p=0$, in (2.26), yields (2.8). Making use of (2.8) in (2.26), we obtain

$$
g(p)=b_{2}(p)+g(0)
$$

for all $p \in I$. From (2.8) and (2.27), it follows that

$$
\sum_{i=1}^{n} g\left(p_{i}\right)=0
$$

and

$$
\sum_{i=1}^{n} \sum_{j=1}^{m} g\left(p_{i} q_{j}\right)=n(m-1) g(0)
$$

hold for all $\left(p_{1}, \ldots, p_{n}\right) \in \Gamma_{n},\left(q_{1}, \ldots, q_{m}\right) \in \Gamma_{m}$. From (1.5), (1.6), (2.25), (2.28) and (2.29), we arrive at the following conclusion:

(2.30) $H: I \rightarrow \mathbb{R}$ is an arbitrary real-valued mapping satisfying (1.6) .

The statement (2.30), together with equations (2.27) and (2.8), constitute the solution (2.7), of (1.5), subject to (1.6).

CASE 2.2. $\lambda \neq 0$.

In this case, let us write (2.24) in the form

$$
\sum_{t=1}^{m}\left[g\left(r_{t}\right)-\lambda H\left(r_{t}\right)\right]=(m-n) g(0)-\lambda .
$$

By Result 1 , there exists an additive mapping $\bar{A}: \mathbb{R} \rightarrow \mathbb{R}$ such that

(2.31) $g(p)-\lambda H(p)=\bar{A}(p)-\frac{1}{m} \bar{A}(1)+\frac{1}{m}\{(m-n) g(0)-\lambda\}$

for all $p \in I$. The substitution $p=0$, in (2.31), gives

$$
\bar{A}(1)=m \lambda H(0)-n g(0)-\lambda .
$$

From (2.31) and (2.32), it follows that

$$
g(p)=\lambda[H(p)-H(0)]+\bar{A}(p)+g(0)
$$


for all $p \in I$. From (2.33) and (2.32), the following equations valid for all $\left(p_{1}, \ldots, p_{n}\right) \in \Gamma_{n}$ and $\left(q_{1}, \ldots, q_{m}\right) \in \Gamma_{m}$ can be derived:

$$
\begin{aligned}
\sum_{i=1}^{n} \sum_{j=1}^{m} g\left(p_{i} q_{j}\right)= & \lambda \sum_{i=1}^{n} \sum_{j=1}^{m} H\left(p_{i} q_{j}\right)-\lambda m(n-1) H(0) \\
& +n(m-1) g(0)-\lambda \\
\sum_{i=1}^{n} g\left(p_{i}\right)= & \lambda \sum_{i=1}^{n} H\left(p_{i}\right)+\lambda(m-n) H(0)-\lambda \\
\sum_{j=1}^{m} g\left(q_{j}\right)= & \lambda \sum_{j=1}^{m} H\left(q_{j}\right)+(m-n) g(0)-\lambda .
\end{aligned}
$$

Also, from (1.6), (2.32) and (2.33), it follows that

$$
g(1)+(n-1) g(0)=0 .
$$

Now, from (1.5), (2.34), (2.35), (2.36), (2.37) and the fact that $\lambda \neq 0$, it follows that

$$
\begin{aligned}
\sum_{i=1}^{n} \sum_{j=1}^{m} H\left(p_{i} q_{j}\right)= & \sum_{i=1}^{n} H\left(p_{i}\right) \sum_{j=1}^{m} H\left(q_{j}\right)+(m-n) H(0) \\
& \times \sum_{j=1}^{m} H\left(q_{j}\right)+m(n-1) H(0) .
\end{aligned}
$$

Thus, we see that $H$ also satisfies the functional equation (1.3). So, by Result 3 , keeping in view (1.6), $H$ is of the form

$$
H(p)=\bar{a}(p)+H(0)
$$

for all $p \in I$, where $\bar{a}: \mathbb{R} \rightarrow \mathbb{R}$ is an additive mapping with

$$
\bar{a}(1)=1-m H(0)
$$

or

$$
H(p)=M(p)-b(p)+H(0)
$$

for all $p \in I$ with $b(1)$ given by (2.10) and $M: I \rightarrow \mathbb{R}$ being a nonconstant nonadditive mapping which is also multiplicative in the sense of Definition 2.1. In the former case, making use of the additivity of $\bar{a}: \mathbb{R} \rightarrow \mathbb{R}$ and (2.40), it follows that $\sum_{j=1}^{m} H\left(q_{j}\right)-1=0$ for all $\left(q_{1}, \ldots, q_{m}\right) \in \Gamma_{m}$, thereby, contradicting (2.23). Hence we ignore (2.39). In the later case, we prove that $\sum_{j=1}^{m} H\left(q_{j}\right)-1$ does not vanish identically on $\Gamma_{m}$. To the contrary, suppose that (2.17) holds 
for all $\left(q_{1}, \ldots, q_{m}\right) \in \Gamma_{m}$. Also, from (2.41), (2.10) and the additivity of $b: \mathbb{R} \rightarrow \mathbb{R}$, we have

$$
\sum_{j=1}^{m} H\left(q_{j}\right)=\sum_{j=1}^{m} M\left(q_{j}\right)
$$

for all $\left(q_{1}, \ldots, q_{m}\right) \in \Gamma_{m}$. Consequently,

$$
\sum_{j=1}^{m} M\left(q_{j}\right)=1
$$

for all $\left(q_{1}, \ldots, q_{m}\right) \in \Gamma_{m}$. By Result 1, there exists an additive mapping $\bar{B}: \mathbb{R} \rightarrow \mathbb{R}$ such that

$$
M(p)=\bar{B}(p)-\frac{1}{m} \bar{B}(1)+\frac{1}{m}
$$

for all $p \in I$. The substitution $p=0$, in (2.42), gives $\bar{B}(1)=1$ as $\bar{B}(0)=0$ and $M(0)=0$. Hence

$$
M(p)=\bar{B}(p)
$$

for all $p \in I$. This contradicts the fact that $M$ is nonadditive. Thus, $\sum_{j=1}^{m} H\left(q_{j}\right)-1$ does not vanish identically on $\Gamma_{m}$. Making use of (2.10) and the fact that $M(1)=1,(1.6)$ follows from (2.41). So, we accept (2.41) with $b(1)=m H(0)$. Now, from (2.33) and (2.41), it follows that

$$
g(p)=\lambda M(p)-\lambda b(p)+\bar{A}(p)+g(0)
$$

for all $p \in I$ with $\lambda \neq 0$. Let us define a mapping $b_{3}: \mathbb{R} \rightarrow \mathbb{R}$ as

$$
b_{3}(p)=-\lambda b(p)+\bar{A}(p)
$$

for all $p \in \mathbb{R}$. Then $b_{3}: \mathbb{R} \rightarrow \mathbb{R}$ is additive. Also, from (2.44), (2.10) and (2.32), it follows that

$$
\lambda=-\left[b_{3}(1)+n g(0)\right] .
$$

Now, from (2.43), (2.44), (2.45) and the fact that $\lambda \neq 0$, it follows that

(2.46) $g(p)=-\left[b_{3}(1)+n g(0)\right] M(p)+b_{3}(p)+g(0), \quad b_{3}(1)+n g(0) \neq 0$

for all $p \in I$. Equations (2.46) and (2.41), together with (2.10), constitute the solution (2.9) of (1.5). This completes the proof of Theorem 2.2.

Note: If we put $p=0$ in (2.18) and make use of the fact that $b_{1}(0)=0$, we get

$$
b_{1}(1)=1-m H(0)
$$


Now we prove:

TheOREM 3.1. Let $n \geq 3, m \geq 3$ be fixed integers and $f: I \rightarrow \mathbb{R}$, $g: I \rightarrow \mathbb{R}, h: I \rightarrow \mathbb{R}, I=[0,1]$, be mappings which satisfy the functional equation (1.4) for all $\left(p_{1}, \ldots, p_{n}\right) \in \Gamma_{n}$ and $\left(q_{1}, \ldots, q_{m}\right) \in \Gamma_{m}$. Then, any general solution of (1.4) is of the form

$$
\left\{\begin{array}{l}
f(p)=a_{1}(p) \\
g(p)=b_{2}(p)+g(0) \\
h \text { an arbitrary mapping }
\end{array}\right.
$$

with $b_{2}(1)$ given by (2.8) or

$$
\left\{\begin{array}{l}
f(p)=a_{1}(p) \\
g \text { an arbitrary mapping } \\
h(p)=a_{2}(p)+h(0)
\end{array}\right.
$$

with

$$
a_{2}(1)=-m h(0)
$$

or

$$
\left\{\begin{array}{rlrl}
f(p)=[h(1)+(m-1) h(0)]\{[g(1)-g(0)] p+a(p)+D(p, p)\} & \\
& +B(p)+f(0) & & \text { if } 0<p \leq 1 \\
= & f(0) & & \text { if } p=0 \\
g(p) & g(0)+[g(1)-g(0)] p+a(p)+D(p, p) & & \text { if } 0<p \leq 1 \\
= & g(0) & & \text { if } p=0 \\
h(p)=[h(1)+(m-1) h(0)]\left[b_{1}(p)-\frac{1}{m} b_{1}(1)+\frac{1}{m}\right]
\end{array}\right.
$$

with

(3.5) $m(n-1) f(0)=[g(1)+(n-1) g(0)][h(1)+(m-1) h(0)]$ and $a: \mathbb{R} \rightarrow \mathbb{R}, D: \mathbb{R} \times] 0,1] \rightarrow \mathbb{R}$ as described in Result 2 or

$$
\left\{\begin{array}{c}
f(p)=[h(1)+(m-1) h(0)]\left\{-\left[b_{3}(1)+n g(0)\right] M(p)+b_{3}(p)\right\}+B(p), \\
{\left[b_{3}(1)+n g(0)\right] \neq 0} \\
g(p)=-\left[b_{3}(1)+n g(0)\right] M(p)+b_{3}(p)+g(0), \\
h(p)=[h(1)+(m-1) h(0)][M(p)-b(p)]+h(0)
\end{array}\right.
$$

with

$$
b(1)=m h(0)[h(1)+(m-1) h(0)]^{-1}
$$

where $[h(1)+(m-1) h(0)] \neq 0$ in (3.4), (3.5), (3.6) and (3.7); $b: \mathbb{R} \rightarrow \mathbb{R}$, $B: \mathbb{R} \rightarrow \mathbb{R}, b_{i}: \mathbb{R} \rightarrow \mathbb{R}(i=1,2,3), a_{i}: \mathbb{R} \rightarrow \mathbb{R}(i=1,2)$ are additive 
mappings; and $M: I \rightarrow \mathbb{R}$ is a nonconstant nonadditive mapping which is multiplicative in the sense of Definition 2.1.

To prove this theorem, we need to prove some lemmas:

LEMmA 3.2. Let $n \geq 3, m \geq 3$ be fixed integers and $f: I \rightarrow \mathbb{R}$ be a mapping which satisfies the functional equation

$$
\sum_{i=1}^{n} \sum_{j=1}^{m} f\left(p_{i} q_{j}\right)=\sum_{j=1}^{m} f\left(q_{j}\right)
$$

for all $\left(p_{1}, \ldots, p_{n}\right) \in \Gamma_{n},\left(q_{1}, \ldots, q_{m}\right) \in \Gamma_{m}$. Then there exists an additive mapping $a_{1}: \mathbb{R} \rightarrow \mathbb{R}$ such that

$$
f(p)=a_{1}(p)
$$

for all $p \in I$.

Proof. Choose $p_{1}=1, p_{2}=\ldots=p_{n}=0 ; q_{1}=1, q_{2}=\ldots=q_{m}=0$ in (3.8). We obtain

$$
m(n-1) f(0)=0 .
$$

Since $m \geq 3, n \geq 3$ are fixed integers, so (3.10) gives

$$
f(0)=0 .
$$

Now putting $q_{1}=1, q_{2}=\ldots=q_{m}=0$ in (3.8) and utilizing (3.11), we get the equation

$$
\sum_{i=1}^{n} f\left(p_{i}\right)=f(1)
$$

valid for all $\left(p_{1}, \ldots, p_{n}\right) \in \Gamma_{n}$. By Result 1 , there exists an additive mapping $a_{1}: \mathbb{R} \rightarrow \mathbb{R}$ such that

$$
f(p)=a_{1}(p)-\frac{1}{n} a_{1}(1)+\frac{1}{n} f(1)
$$

for all $p \in I$. Putting $p=0$ in (3.12), using (3.11) and the fact that $a_{1}(0)=0$, it follows that $a_{1}(1)=f(1)$. Now, (3.12) reduces to (3.9).

LEMMA 3.3. Under the conditions stated in the statement of Theorem 3.1, the equation

$$
\begin{aligned}
& {[h(1)+(m-1) h(0)] \sum_{i=1}^{n} \sum_{j=1}^{m} g\left(p_{i} q_{j}\right)} \\
& =\sum_{i=1}^{n} g\left(p_{i}\right) \sum_{j=1}^{m} h\left(q_{j}\right)+[h(1)+(m-1) h(0)] \sum_{j=1}^{m} g\left(q_{j}\right) \\
& \quad+m(n-1)[h(1)+(m-1) h(0)] g(0) \\
& \quad-[g(1)+(n-1) g(0)][h(1)+(m-1) h(0)]
\end{aligned}
$$


holds.

Proof. Let us choose $q_{1}=1, q_{2}=\ldots=q_{m}=0$ in (1.4). We obtain the equation

$$
\begin{aligned}
& \sum_{i=1}^{n}\left\{f\left(p_{i}\right)-[h(1)+(m-1) h(0)] g\left(p_{i}\right)\right\} \\
& =f(1)-(n-1)(m-1) f(0) .
\end{aligned}
$$

By Result 1 , there exists an additive mapping $B: \mathbb{R} \rightarrow \mathbb{R}$ such that

$$
\begin{aligned}
f(p) & -[h(1)+(m-1) h(0)] g(p) \\
& =B(p)-\frac{1}{n} B(1)+\frac{1}{n}\{f(1)-(n-1)(m-1) f(0)\}
\end{aligned}
$$

for all $p \in I$. Substituting $p=0$ and using the fact that $B(0)=0,(3.15)$ gives

(3.16) $B(1)=[h(1)+(m-1) h(0)] n g(0)+f(1)-(n m-m+1) f(0)$.

From (3.15) and (3.16), it follows that

(3.17) $\quad f(p)=[h(1)+(m-1) h(0)][g(p)-g(0)]+B(p)+f(0)$

for all $p \in I$. From (3.17) and (3.16), the following two equations valid for all $\left(p_{1}, \ldots, p_{n}\right) \in \Gamma_{n}$ and $\left(q_{1}, \ldots, q_{m}\right) \in \Gamma_{m}$ can be obtained:

$$
\begin{aligned}
\sum_{i=1}^{n} \sum_{j=1}^{m} f\left(p_{i} q_{j}\right)= & {[h(1)+(m-1) h(0)] \sum_{i=1}^{n} \sum_{j=1}^{m} g\left(p_{i} q_{j}\right) } \\
& -n(m-1) g(0)[h(1)+(m-1) h(0)] \\
& +f(1)+(m-1) f(0), \\
\sum_{j=1}^{m} f\left(q_{j}\right)= & {[h(1)+(m-1) h(0)] \sum_{j=1}^{m} g\left(q_{j}\right) } \\
& +(n-m)[h(1)+(m-1) h(0)] g(0) \\
& +f(1)-(n m-2 m+1) f(0) .
\end{aligned}
$$

From (1.4), (3.18) and (3.19), it follows that

$$
\begin{aligned}
{[h(1)} & +(m-1) h(0)] \sum_{i=1}^{n} \sum_{j=1}^{m} g\left(p_{i} q_{j}\right) \\
= & \sum_{i=1}^{n} g\left(p_{i}\right) \sum_{j=1}^{m} h\left(q_{j}\right)+[h(1)+(m-1) h(0)] \sum_{j=1}^{m} g\left(q_{j}\right) \\
& +m(n-1) g(0)[h(1)+(m-1) h(0)]-m(n-1) f(0) .
\end{aligned}
$$

Now let us put $p_{1}=1, p_{2}=\ldots=p_{n}=0$ in (3.14). We obtain (3.5). From (3.20) and (3.5), the required equation (3.13) follows immediately. 
Proof of Theorem 3.1. We divide our discussion into three cases.

CASE 1. $\sum_{i=1}^{n} g\left(p_{i}\right)$ vanishes identically on $\Gamma_{n}$, that is, (2.28) holds for all $\left(p_{1}, \ldots, p_{n}\right) \in \Gamma_{n}$. Then, (1.4) reduces to (3.8) and $h$ can be an arbitrary mapping. By Result 1, applied to (2.28), there exists an additive mapping $b_{2}: \mathbb{R} \rightarrow \mathbb{R}$ such that $g(p)$ is of the form (2.27) with $b_{2}(1)$ given by (2.8). Also, by Lemma $3.2, f$ is of the form (3.9) in which $a_{1}: \mathbb{R} \rightarrow \mathbb{R}$ is an additive mapping. Thus, we have obtained the solution (3.1) of (1.4) with $b_{2}(1)$ given by $(2.8)$.

CASE 2. $\sum_{j=1}^{m} h\left(q_{j}\right)$ vanishes identically on $\Gamma_{m}$. In this case, solution (3.2) follows. The details are omitted as the discussion is similar to the one in Case 1.

CASE 3. Neither $\sum_{i=1}^{n} g\left(p_{i}\right)$ vanishes identically on $\Gamma_{n}$ nor $\sum_{j=1}^{m} h\left(q_{j}\right)$ vanishes identically on $\Gamma_{m}$. Then there exist a $\left(p_{1}^{*}, \ldots, p_{n}^{*}\right) \in \Gamma_{n}$ and a $\left(q_{1}^{*}, \ldots, q_{m}^{*}\right) \in$ $\Gamma_{m}$ such that $\sum_{i=1}^{n} g\left(p_{i}^{*}\right) \neq 0$ and $\sum_{j=1}^{m} h\left(q_{j}^{*}\right) \neq 0$ and consequently

$$
\sum_{i=1}^{n} g\left(p_{i}^{*}\right) \sum_{j=1}^{m} h\left(q_{j}^{*}\right) \neq 0 .
$$

We prove that $[h(1)+(m-1) h(0)] \neq 0$. To the contrary, suppose that $h(1)+(m-1) h(0)=0$. Then equation (3.13) reduces to

$$
\sum_{i=1}^{n} g\left(p_{i}\right) \sum_{j=1}^{m} h\left(q_{j}\right)=0
$$

valid for all $\left(p_{1}, \ldots, p_{n}\right) \in \Gamma_{n}$ and $\left(q_{1}, \ldots, q_{m}\right) \in \Gamma_{m}$. In particular, $\sum_{i=1}^{n} g\left(p_{i}^{*}\right)$ $\times \sum_{j=1}^{m} h\left(q_{j}^{*}\right)=0$ thereby contradicting $(3.21)$. Hence $h(1)+(m-1) h(0) \neq 0$. Dividing both sides of $(3.13)$ by $[h(1)+(m-1) h(0)]$, we obtain

$$
\begin{aligned}
\sum_{i=1}^{n} \sum_{j=1}^{m} g\left(p_{i} q_{j}\right)= & \sum_{i=1}^{n} g\left(p_{i}\right)\left(\frac{\sum_{j=1}^{m} h\left(q_{j}\right)}{h(1)+(m-1) h(0)}\right) \\
& +\sum_{j=1}^{m} g\left(q_{j}\right)-[g(1)-(n-1)(m-1) g(0)]
\end{aligned}
$$

Define a mapping $H: I \rightarrow \mathbb{R}$ as

$$
H(x)=[h(1)+(m-1) h(0)]^{-1} h(x)
$$


for all $x \in I$. Then, equation (3.22) reduces to the functional equation (1.5). Moreover, (3.23) gives (1.6). Now we can make use of Theorem 2.2.

The solution (3.4), of (1.4), follows from (2.6), (3.17) and (3.23).

To proceed further, we observe that, from (2.7) and (2.8), (2.37) follows. Making use of it in (3.5), we get $f(0)=0$. Now equations (2.7), (3.17) and (3.23) give rise to the solution

$$
\left\{\begin{array}{l}
f(p)=[h(1)+(m-1) h(0)] b_{2}(p)+B(p) \\
g(p)=b_{2}(p)+g(0) \\
h \text { an arbitrary mapping with } h(1)+(m-1) h(0) \neq 0
\end{array}\right.
$$

of (1.4) along with (2.8). But this solution is included in (3.1).

Equation (2.37) also follows from (2.9) and the fact that $M(1)=1$. Hence, as above, we again have $f(0)=0$. The solution (3.6), with $b(1)$ given by (3.7), now follows from (2.9), (3.17), (2.10) and (3.23). This completes the proof of Theorem 3.1.

Note: from (2.47) and (3.23), it follows that

$$
b_{1}(1)=\frac{h(1)-h(0)}{h(1)+(m-1) h(0)} .
$$

\section{Comments}

The object of this section is to comment upon the various solutions of Theorem 3.1 from the point of view of information theory.

The solution (3.1) is not of any relevance in information theory as the mapping $h$ in it is arbitrary and the summands $\sum_{i=1}^{n} f\left(p_{i}\right)$ and $\sum_{i=1}^{n} g\left(p_{i}\right)$ are independent of the probabilities $p_{1}, \ldots, p_{n}$. Similarly, the solution (3.2) is also not of any relevance in information theory. Now we discuss the importance of solution (3.4) in information theory.

Let $\left(p_{1}, \ldots, p_{n}\right) \in \Gamma_{n},\left(q_{1}, \ldots, q_{m}\right) \in \Gamma_{m}, n \geq 3, m \geq 3$ being fixed integers. Let

$$
S_{1}=\left\{i: 0<p_{i} \leq 1,1 \leq i \leq n\right\} \text { and } S_{2}=\left\{j: 0<q_{j} \leq 1,1 \leq j \leq m\right\} .
$$

Then $S_{1}$ and $S_{2}$ are nonempty sets. Let $n_{0}$ and $m_{0}$ denote respectively the number of elements in $S_{1}$ and $S_{2}$. Then $1 \leq n_{0} \leq n$ and $1 \leq m_{0} \leq m$. Making use of the additivity of $\left.\left.a: \mathbb{R} \rightarrow \mathbb{R}, B: \mathbb{R} \rightarrow \mathbb{R}, b_{1}: \mathbb{R} \rightarrow \mathbb{R}, D: \mathbb{R} \times\right] 0,1\right] \rightarrow \mathbb{R}$ (in the first variable), (2.4), (2.5), (3.24) and the fact that $a(1)=E(1,1)$, 
from (3.4), it follows that

$$
\begin{aligned}
\sum_{i=1}^{n} \sum_{j=1}^{m} f\left(p_{i} q_{j}\right)= & n_{0} m_{0} f(0)+[h(1)+(m-1) h(0)] \\
& \times\left\{g(1)-g(0)-D(1,1)+\sum_{i \in S_{1}} \sum_{j \in S_{2}} D\left(p_{i} q_{j}, p_{i} q_{j}\right)\right\} \\
& +B(1)+\left(m n-m_{0} n_{0}\right) f(0)
\end{aligned}
$$

or

$$
\text { (4.1) } \begin{aligned}
\sum_{i=1}^{n} \sum_{j=1}^{m} f\left(p_{i} q_{j}\right)= & n m f(0)+[h(1)+(m-1) h(0)] \\
\times & \left\{g(1)-g(0)-2 D(1,1)+\sum_{i \in S_{1}} D\left(p_{i}, p_{i}\right)\right. \\
& \left.+\sum_{j \in S_{2}} D\left(q_{j}, q_{j}\right)\right\}+B(1) .
\end{aligned}
$$

Also

$$
\begin{aligned}
\sum_{i=1}^{n} g\left(p_{i}\right)= & n_{0} g(0)+[g(1)-g(0)]-D(1,1) \\
& +\sum_{i \in S_{1}} D\left(p_{i}, p_{i}\right)+\left(n-n_{0}\right) g(0)
\end{aligned}
$$

or

(4.2) $\sum_{i=1}^{n} g\left(p_{i}\right)=[g(1)+(n-1) g(0)]-D(1,1)+\sum_{i \in S_{1}} D\left(p_{i}, p_{i}\right)$.

Moreover,

$$
\sum_{j=1}^{m} h\left(q_{j}\right)=h(1)+(m-1) h(0)
$$

and

$$
\begin{aligned}
\sum_{j=1}^{m} f\left(q_{j}\right)= & m_{0} f(0)+[h(1)+(m-1) h(0)]\{g(1)-g(0) \\
& \left.-D(1,1)+\sum_{j \in S_{2}} D\left(q_{j}, q_{j}\right)\right\}+B(1)+\left(m-m_{0}\right) f(0)
\end{aligned}
$$


or

$$
\text { (4.4) } \begin{aligned}
\sum_{j=1}^{m} f\left(q_{j}\right)= & m f(0)+[h(1)+(m-1) h(0)] \\
& \times\left\{g(1)-g(0)-D(1,1)+\sum_{j \in S_{2}} D\left(q_{j}, q_{j}\right)\right\}+B(1) .
\end{aligned}
$$

Making use of the equations (4.1), (4.2), (4.3) and (4.4), it can be seen that (1.4) is satisfied if (3.5) holds. To proceed further, we also need

$$
\begin{aligned}
\sum_{i=1}^{n} f\left(p_{i}\right)= & n f(0)+[h(1)+(m-1) h(0)] \\
& \times\left\{g(1)-g(0)-D(1,1)+\sum_{i \in S_{1}} D\left(p_{i}, p_{i}\right)\right\}+B(1) \\
\sum_{i=1}^{n} h\left(p_{i}\right)= & {[h(1)+(m-1) h(0)]\left[\frac{m-n}{m} b_{1}(1)+\frac{n}{m}\right] . }
\end{aligned}
$$

Keeping in view the form of the Shannon entropy given by $(\mathrm{A})$, it seems desirable to choose the mapping $D: \mathbb{R} \times] 0,1] \rightarrow \mathbb{R}$ defined as

$$
D(x, y)=c x \log _{2} y
$$

for all $x \in \mathbb{R}, y \in] 0,1], c$ an arbitrary real constant. The case $c=0$ is not of much importance. So we restrict to $c \neq 0$. Now

$$
D(p, p)=c p \log _{2} p
$$

for all $p \in] 0,1]$ and $D(1,1)=0$. To accommodate the 0 -probabilities, we may assume

$$
\lim _{p \rightarrow 0^{+}} D(p, p)=0
$$

or equivalently $0 \log _{2} 0=0$ as $c \neq 0$.

The summand $\sum_{i=1}^{n} h\left(p_{i}\right)$, in (4.6), is independent of the probabilities $p_{1}, \ldots, p_{n}$. Hence it seems to be of no use. But, (4.5) and (4.2), together with (4.8), (A), $D(1,1)=0$ and $0 \log _{2} 0=0$, give

$$
\begin{aligned}
\sum_{i=1}^{n} f\left(p_{i}\right)= & n f(0)+[h(1)+(m-1) h(0)] \\
& \times\left\{g(1)-g(0)-c H_{n}\left(p_{1}, \ldots, p_{n}\right)\right\}+B(1) \\
\sum_{i=1}^{n} g\left(p_{i}\right)= & {[g(1)+(n-1) g(0)]-c H_{n}\left(p_{1}, \ldots, p_{n}\right) . }
\end{aligned}
$$

Thus, we see that the mappings $f$ and $g$, appearing in (1.4), are related to the Shannon entropy. 
It may be noted that the mapping $D: \mathbb{R} \times] 0,1] \rightarrow \mathbb{R}$, defined by (4.7), is additive in the first variable and satisfies $(2.4)$ as $E(1,1)=a(1)=-D(1,1)=0$.

Now we discuss the solution (3.6), together with (3.7). Making use of the additivity of the mappings $b_{3}: \mathbb{R} \rightarrow \mathbb{R}, b: \mathbb{R} \rightarrow \mathbb{R}, B: \mathbb{R} \rightarrow \mathbb{R}$; the multiplicativity of $M: I \rightarrow \mathbb{R}$ in the sense of Definition 2.1, it follows that

$$
\begin{aligned}
\sum_{i=1}^{n} \sum_{j=1}^{m} f\left(p_{i} q_{j}\right)= & {[h(1)+(m-1) h(0)]\left[b_{3}(1)+n g(0)\right] } \\
& \times\left[1-\sum_{i=1}^{n} M\left(p_{i}\right) \sum_{j=1}^{m} M\left(q_{j}\right)\right] \\
& -n g(0)[h(1)+(m-1) h(0)]+B(1), \\
\sum_{i=1}^{n} g\left(p_{i}\right)= & {\left[b_{3}(1)+n g(0)\right]\left[1-\sum_{i=1}^{n} M\left(p_{i}\right)\right], } \\
\sum_{j=1}^{m} h\left(q_{j}\right)= & {[h(1)+(m-1) h(0)] \sum_{j=1}^{m} M\left(q_{j}\right), } \\
\sum_{j=1}^{m} f\left(q_{j}\right)= & {[h(1)+(m-1) h(0)]\left[b_{3}(1)+n g(0)\right] } \\
& \times\left[1-\sum_{j=1}^{m} M\left(q_{j}\right)\right] \\
& -n g(0)[h(1)+(m-1) h(0)]+B(1) .
\end{aligned}
$$

It can be verified that (4.10) to (4.13) do satisfy (1.4). To proceed further, we need

$$
\begin{aligned}
& \sum_{i=1}^{n} f\left(p_{i}\right)=[h(1)+(m-1) h(0)]\left[b_{3}(1)+n g(0)\right] \\
& \times\left[1-\sum_{i=1}^{n} M\left(p_{i}\right)\right]-n g(0)[h(1)+(m-1) h(0)]+B(1), \\
& \text { (4.15) } \sum_{i=1}^{n} h\left(p_{i}\right)=[h(1)+(n-1) h(0)]-[h(1)+(m-1) h(0)] \\
& \times\left[1-\sum_{i=1}^{n} M\left(p_{i}\right)\right] \text {. }
\end{aligned}
$$

From the point of view of information theory, taking into consideration the nonadditive measure of entropy given by Havrda and Charvát [2] given by (B), it is desirable to choose the mapping $M: I \rightarrow \mathbb{R}$ defined as $M(p)=p^{\alpha}$, 
$0 \leq p \leq 1, \alpha \in \mathbb{R}, \alpha>0, \alpha \neq 1,0^{\alpha}:=0$ and $1^{\alpha}:=1$. Then, we get

$$
\begin{aligned}
\sum_{i=1}^{n} f\left(p_{i}\right)= & {[h(1)+(m-1) h(0)]\left[b_{3}(1)+n g(0)\right] } \\
& \times\left(1-2^{1-\alpha}\right) H_{n}^{\alpha}\left(p_{1}, \ldots, p_{n}\right) \\
& -n g(0)[h(1)+(m-1) h(0)]+B(1), \\
\sum_{i=1}^{n} g\left(p_{i}\right)= & {\left[b_{3}(1)+n g(0)\right]\left(1-2^{1-\alpha}\right) H_{n}^{\alpha}\left(p_{1}, \ldots, p_{n}\right), } \\
\sum_{i=1}^{n} h\left(p_{i}\right)= & {[h(1)+(n-1) h(0)]-[h(1)+(m-1) h(0)] } \\
& \times\left(1-2^{1-\alpha}\right) H_{n}^{\alpha}\left(p_{1}, \ldots, p_{n}\right) .
\end{aligned}
$$

Thus we see that all the three mappings $f, g$ and $h$, appearing in (1.4), are related to the nonadditive entropy of degree $\alpha$ due to Havrda and Charvát [2].

Losonczi and Maksa [3] characterized the entropies

$$
L_{n}^{\mu}\left(p_{1}, \ldots, p_{n}\right)=\frac{1}{\mu}\left[\sum_{i=1}^{n} M\left(p_{i}\right)-1\right]
$$

where $\mu \neq 0$.

If we use (4.16), then (4.14), (4.11) and (4.15) can be written respectively in the form

$$
\begin{aligned}
\sum_{i=1}^{n} f\left(p_{i}\right)= & -[h(1)+(m-1) h(0)]\left[b_{3}(1)+n g(0)\right] \mu L_{n}^{\mu}\left(p_{1}, \ldots, p_{n}\right) \\
& -n g(0)[h(1)+(m-1) h(0)]+B(1) \\
\sum_{i=1}^{n} g\left(p_{i}\right)= & -\left[b_{3}(1)+n g(0)\right] \mu L_{n}^{\mu}\left(p_{1}, \ldots, p_{n}\right), \\
\sum_{i=1}^{n} h\left(p_{i}\right)= & {[h(1)+(n-1) h(0)]+[h(1)+(m-1) h(0)] \mu L_{n}^{\mu}\left(p_{1}, \ldots, p_{n}\right) . }
\end{aligned}
$$

\section{REFERENCES}

[1] Z. Daróczy and L. Losonczi, Über die Erweiterung der auf einer Punktmenge additiven Funktionen, Publ. Math. 14 (1967), 239-245 (in German).

[2] J. Havrda and F. Charvát, Quantification method of classification process, concept of structural $\alpha$-entorpy, Kybernetika 3 (1967), 30-35.

[3] L. Losonczi and Gy. Maksa, On some functional equations of the information theory, Acta Math. Acad. Sci. Hungar. 39 (1982), 73-82.

[4] P. Nath and D. K. Singh, On a multiplicative type sum form functional equation and its role in information theory, Applications of Mathematics 51(5) (2006), 495-516.

[5] C. E. Shannon, A mathematical theory of communication, Bell Syst. Tech. Jour. 27 (1948), 378-423, 623-656 
P. Nath

Department of Mathematics

University of Delhi

Delhi 110007

India

E-mail: pnathmaths@gmail.com

D. K. Singh

Department of Mathematics

University of Delhi

Delhi 110007

India

E-mail: dksingh@maths.du.ac.in

dhiraj426@rediffmail.com

Received: 16.2.2007. 